\title{
Kopfschmerzen bei Hirntumoren
}

\section{S. Evers}

Klinik für Neurologie, Krankenhaus Lindenbrunn, Coppenbrügge

\begin{abstract}
Schlüsselwörter
Hirntumor, Kopfschmerzen, Hypophysenadenom, Opioide

Zusammenfassung

Kopfschmerzen sind für viele Patienten Anlass, an einen Hirntumor zu denken. Dabei sind Kopfschmerzen bei Hirntumoren als Erstsymptom selten. Im Lauf einer Hirntumorerkrankung leider aber doch bis zu ca. $80 \%$ aller Patienten an Kopfschmerzen. Am häufigsten treten Kopfschmerzen bei Tumoren in der Sellaregion und bei Infiltration schmerzsensibler Strukturen wie Meningen und bestimmte Hirnnerven auf. Hirntumore, insbesondere Hypophysenadenome, können auch idiopathische Kopfschmerzen imitieren. Dagegen sind idiopathische Kopfschmerzen kein Risikofaktor für die Entstehung von Hirntumoren. Die Behandlung der Kopfschmerzen bei Hirntumoren ist unspezifisch und schließt auch Opioide und Steroide mit ein.
\end{abstract}

\section{Keywords}

Brain tumor, headache, pituitary adenoma, opioids

\section{Summary}

Headache makes many patients fear a brain tumor. However, headache is only very rarely primary symptom of a brain tumor. In the course of brain tumors, up to $80 \%$ of all patients suffer from headache. The most frequent brain tumors causing headache are tumors of the sella region and infiltration of pain sensitive structures such as the meninges and some cranial nerves. Brain tumors, in particular pituitary adenomas, can mimic idiopathic headaches. In contrast, idiopathic headaches are not a risk factor for the development of brain tumors. The treatment of headache in brain tumors is unspecific and encompasses also opioids and steroids.

Korrespondenzadresse

Prof. Dr. med. Dr. phil. Stefan Evers

Klinik für Neurologie

Krankenhaus Lindenbrunn

Lindenbrunn 1, 31863 Coppenbrügge

everss@uni-muenster.de
Headache in brain tumors

Nervenheilkunde 2018; 37: 53-58

eingegangen am: 20. September 2017

angenommen am: 4. Oktober 2017
Kopfschmerzen bei Hirntumoren stellen ein im Vergleich zu den idiopathischen und anderen symptomatischen Kopfschmerzen extrem seltenes Phänomen bezogen auf die Gesamtbevölkerung dar. In starkem Kontrast dazu besteht jedoch bei fast jedem Kopfschmerz-Patienten eine Tumorangst, die der häufigste Anlass für zerebrale Bildgebung, ist. Kopfschmerzen sind jedoch bei unter $1 \%$ aller Kopfschmerzpatienten das erste Symptom eines Hirntumors. Zur Verdeutlichung mögen folgende Zahlen dienen (1): Von 10000 Menschen haben 8000 im vergangenen Jahr Kopfschmerzen gehabt, davon 1000 mit wenigstens einem Anfall starker Kopf- schmerzen. 160 von diesen Patienten gehen wegen ihrer Kopfschmerzen zum Arzt, 20 werden wegen ihrer Kopfschmerzen stationär behandelt; und nur bei einem von diesen 10000 Menschen wird im Lauf des nächsten Jahres ein Hirntumor diagnostiziert, der zudem nur mit einer Wahrscheinlichkeit von ca. $60 \%$ auch zu Kopfschmerzen geführt hat.

Im folgenden Text (Überarbeitung nach 2) soll es um die verschiedenen Häufigkeiten und Entstehungsmechanismen, die besonderen klinischen Erscheinungsbilder und die möglichen spezifischen Therapieverfahren bei Kopfschmerzen aufgrund von Hirntumoren inklusive intrakranieller
Metastasen und der Meningeosis carcinomatosa gehen. Dabei sind nur sehr wenig Originalarbeiten über Kopfschmerzen aufgrund von Hirntumoren publiziert (3).

\section{Epidemiologie}

Die Prävalenz von Kopfschmerzen bei Patienten mit einem Hirntumor ist bislang nur wenig in epidemiologischen Studien untersucht worden. In $>$ Tabelle 1 sind die Ergebnisse der wichtigsten Studien, die sich mit dieser Frage beschäftigt haben, aufgelistet, es handelt sich durchweg um ältere Studien. Zwar schwanken die Prävalenzzahlen erheblich, es lassen sich aber dennoch einige typische Muster ableiten. So sind Kopfschmerzen bei supratentoriellen Hirntumoren seltener als bei infratentoriellen Hirntumoren. Extrazerebrale Tumoren führen seltener zu Kopfschmerzen als intrazerebrale (4); hochmaligne Gliome führen häufiger zu Kopfschmerzen als niedriggradige Astrozytome, die z. B. eher durch epileptische Anfälle als durch Kopfschmerzen symptomatisch werden. Hirntumoren im Bereich der ableitenden Liquorwege führen durch den Liquoraufstau häufiger zu Kopfschmerzen als Tumore, die vom Ventrikelsystem entfernt lokalisiert sind (5). In einer Fallserie von 323 Patienten mit Oligodendrogliom waren Kopfschmerzen das häufigste Symptom noch vor epileptischen Anfällen und Sehstörungen, die Kopfschmerzen zeigten aber keine Korrelation zu dem Malignitätsgrad des Tumors (6). Insgesamt treten Kopfschmerzen bei ca. zwei Drittel aller Patienten mit einem Hirntumor auf.

Die Art des Hirntumors scheint ebenfalls Einfluss auf das Auftreten von Kopfschmerzen zu nehmen. Am häufigsten kommt es zu Kopfschmerzen bei nasopharyngealen Karzinomen, am seltensten bei den Hirnbasistumoren und bei den Meningeomen. \Tabelle 2 gibt eine Übersicht über die Häufigkeit von Kopfschmerzen 


\begin{tabular}{|l|l|l|}
\hline Quelle & Prävalenz (\%) & Anmerkung \\
\hline Rushton et al. (10) & 60 & $\begin{array}{l}\text { 58\% supratentoriell, 64\% infra- } \\
\text { tentoriell }\end{array}$ \\
\hline Iversen et al. (5) & 53 & - \\
\hline CBTC (24) & 62 & $\begin{array}{l}\text { nur Kinder untersucht; 58\% } \\
\text { supratentoriell, 70\% infratentoriell }\end{array}$ \\
\hline Forsyth et al. (14) & 48 & $\begin{array}{l}\text { 40\% supratentoriell, 82\% infra- } \\
\text { tentoriell }\end{array}$ \\
\hline Jaeckle (7) & 60 & - \\
\hline Suwanwela et al. (4) & 72 & nur hirneigene Tumoren \\
\hline Pfund et al. (15) & 59 & nur Hirnmetastasen \\
\hline Schankin et al. (16) & 60 & - \\
\hline Schankin et al. (25) & 41 & - \\
\hline Russo et al. (26) & 13 & Hypophysenadenome \\
\hline Siegel et al. (23) & 53 & nur Gliome \\
\hline
\end{tabular}

bei verschiedenen Hirn- und Schädeltumoren. Hierbei ist zu berücksichtigen, dass die hier aufgeführten Prävalenzzahlen eher eine Überschätzung des Problems darstellen, da viele Tumore erst spät diagnostiziert

Tab. 2 Häufigkeit von Kopfschmerzen bei verschiedenen Formen von Hirn- und Schädeltumoren (nach 3, 9, 27).

\begin{tabular}{|l|l|}
\hline Tumorart & $\begin{array}{l}\text { Häufigkeit von } \\
\text { Kopfschmerzen } \\
(\%)\end{array}$ \\
\hline $\begin{array}{l}\text { Nasopharyngeale } \\
\text { Karzinome }\end{array}$ & 83 \\
\hline $\begin{array}{l}\text { Primäre hirneigene } \\
\text { Tumore }\end{array}$ & $\begin{array}{l}50 \text { bis } 72 \text { (bei } \\
\text { Erwachsenen) }\end{array}$ \\
\hline $\begin{array}{l}\text { Primäre hirneigene } \\
\text { Tumore }\end{array}$ & 64 (bei Kindern) \\
\hline $\begin{array}{l}\text { Parenchymmetastasen } \\
\text { Leptomeningeale } \\
\text { Metastasen/Meningeosis }\end{array}$ & 50 bis 67 \\
\hline $\begin{array}{l}\text { Metastasen der } \\
\text { Schädelbasis }\end{array}$ & 33 bis 76 \\
\hline Hirnstammgliome & 44 \\
\hline Hypophysenadenome & 41 \\
\hline Meningeom & 33 \\
\hline Akustikusneurinome & 30 \\
\hline Neurofibromatose Typ I & 30 \\
\hline $\begin{array}{l}\text { Chordome der } \\
\text { Schädelbasis }\end{array}$ & 27 \\
\hline
\end{tabular}

Tab. 1

Prävalenz von Kopfschmerzen bei Patienten mit einem Hirntumor. werden und bis heute kleinere Metastasen und frühe hirneigene Tumore auch bildgebend nicht sicher dargestellt werden können. Als Richtgröße für einen Tumor, der durch seine Größenwirkung Kopfschmerzen verursacht, werden mindestens $2 \mathrm{~cm}$ Durchmesser angegeben.

Es ist insgesamt eine Ausnahme, dass ein Hirntumor primär und nur durch Kopfschmerzen auffällig wird. Zwar haben $16 \%$ bis $20 \%$ der Patienten mit Hirntumor initial Kopfschmerzen $(5,7)$, doch können dabei meistens noch weitere Symptome in der Untersuchung aufgedeckt werden. In einer Studie an 183 Patienten mit einem Hirntumor hatten nur 8\% Kopfschmerzen als initiales und isoliertes Symptom (8). Am ehesten zeigen noch infratentorielle Hirntumoren, die zu einer Abflussbehinderung des Liquors führen (z. B. Medulloblastome, die zu 90\% mit Kopfschmerzen einhergehen), initiale Kopfschmerzen. Viel häufiger ist es, dass Kopfschmerzen eines von mehreren Begleitsymptomen sind (neben z. B. Erbrechen, Sehstörungen, Schwindel) und dass bei der klinisch-neurologischen Untersuchung fokale Symptome auffallen. Bei den gutartigen Hirntumoren z. B. Neurofibromen, Akustikusneurinomen sind Kopfschmerzen nicht häufiger als in der gesunden Normalbevölkerung (9).

\section{Pathophysiologie}

Zwar imitieren Kopfschmerzen durch Hirntumoren weit überwiegend semiologisch Kopfschmerzen vom Spannungstyp, in selteneren Fällen auch Migräne ohne oder mit Aura, ihre Pathophysiologie muss jedoch von der der idiopathischen Kopfschmerzen unterschieden werden.

Zum einen können Hirntumoren Kopfschmerzen dadurch verursachen, dass sie schmerzsensible Strukturen direkt reizen. Dies trifft z. B. für die Hirnhäute, die gröBeren Arterien und Venen, die sensiblen Hirnnerven in ihren intrakraniellen Abschnitten und das Tentorium zu. Studien zur Übertragung von Schmerzen durch Reizung von intrakraniellen Strukturen konnten zeigen, dass z. B. eine Affektion des vorderen Tentoriums zu frontal und temporal empfundenen Kopfschmerzen führen kann. Reizung in der hinteren Schädelgrube, aber auch der parietalen Meningen, kann zu Schmerzen führen, die im Nacken lokalisiert werden. In seltenen Fällen können hirneigene Tumoren und häufiger noch Metastasen bluten und so zu einer Reizung der Subarachnoidea führen.

Neben dieser direkten Reizung von schmerzsensiblen Strukturen können Hirntumoren Kopfschmerzen durch eine Raumforderung und die damit verbundene Ödembildung oder durch die Behinderung des Liquorabflusses und damit über einen Hydrocephalus internus verursachen. Die genauen Mechanismen dieses Zusammenhangs zwischen der intrakraniellen raumfordernden und druckerhöhenden Wirkung und der Entstehung von Kopfschmerzen ist noch wenig bekannt. Es besteht kein sehr enger Zusammenhang zwischen dem intrakraniellen mechanischen Druck und der Empfindung von Kopfschmerzen. So haben bis zu einem Drittel der Hirntumorpatienten mit einem erhöhten intrakraniellen Druck keine Kopfschmerzen $(10,11)$.

Noch sehr wenig erforscht ist, inwieweit Hirntumoren auch durch inflammatorische oder andere metabolische/katabolische Mechanismen zur Entstehung von Kopfschmerzen beitragen können, wie dies für den Tumorschmerz im allgemeinen bereits seit längerem untersucht und postuliert wird (12). 


\section{Klinisches Bild}

\section{Zeitmuster}

Kopfschmerzen aufgrund von Hirntumoren sind überraschenderweise, aber typischerweise nicht konstant, sondern in drei Viertel aller Fälle intermittierend oder fluktuierend (4). Nur ein Drittel der Patienten mit Kopfschmerzen aufgrund von Hirntumoren hat die ansonsten als typisch angesehenen morgendlichen Kopfschmerzen mit Erbrechen. Bei ca. 20\% erweckt der Kopfschmerz die Patienten morgens aus dem Schlaf. Nächtliche Kopfschmerzen werden in verschiedenen Studien zwischen $32 \%$ und $71 \%$ berichtet. Eine Erhöhung des intrakraniellen Drucks wie etwa beim Valsalva-Manöver führt bei ca. ein Drittel zu einer vorübergehenden Verstärkung der Kopfschmerzen.

\section{Lokalisation}

Die Lokalisation von Kopfschmerzen aufgrund von Hirntumoren kann sehr wechselhaft sein und ist im Allgemeinen unspezifisch. Am häufigsten tritt der Kopfschmerz bifrontal auf, vor allem bei supratentoriellen Tumoren. Eine Lokalisation im okzipitalen oder nuchalen Bereich ist eher mit infratentoriellen Hirntumoren assoziiert. Die engste Korrelation bezüglich der Lokalisation besteht zwischen einer konstanten Halbseitigkeit von Kopfschmerzen und einem dann auch ipsilateralen Hirntumor.

\section{Intensität}

Die Intensität von Kopfschmerzen bei Hirntumoren kann sowohl intra- als auch interindividuell sehr großen Schwankungen unterliegen. In einem großen unselektierten Kollektiv von Hirntumor-Patienten betrug der Median der Schmerzintensität 7 auf einer Skala von 1 bis 10 (3). Eine starke Intensität tritt eher bei Kopfschmerzen auf, die mit einer intrakraniellen Drucksteigerung einhergehen, sie können häufig in ihrer Stärke nicht durch Analgetika beeinflusst werden. Leichtere Kopfschmerzen sind häufiger bei supratentoriellen Hirntumoren ohne Liquorabflussbehinderung, sie werden dann häufig mit einem Kopfschmerz vom Spannungstyp verwechselt, zumal sie eher gut auf Analgetika ansprechen. Das vielfach berichtete morgendliche Intensitätsmaximum weisen nur ca. 20\% aller Hirntumorpatienten mit Kopfschmerzen auf. Eine langsame Progression der Schmerzintensität spricht aufgrund epidemiologischer Studien am ehesten für Hirnmetastasen als Ursache der Kopfschmerzen.

\section{Begleitsymptome}

Nur sehr selten tritt Kopfschmerz als einziges Symptom eines Hirntumors auf. Meistens finden sich zusätzlich Auffälligkeiten in der neurologischen Untersuchung oder vegetative Symptome. In einer Analyse von 1282 Kopfschmerz-Patienten mit einer unauffälligen neurologischen Untersuchung und ohne weitere subjektive Symptome fand sich nur einer mit einem Hirntumor (13). Kopfschmerzen ohne weitere subjektive Symptome treten in ca. $10 \%$ aller Patienten mit Hirntumoren auf; diese bis auf die Kopfschmerzen asymptomatische Phase dauert im Durchschnitt 10 Wochen an, bis dann andere Symptome wie Paresen oder epileptische Anfälle auftreten. Die häufigsten Begleitsymptome bei Kopfschmerzen aufgrund von Hirntumoren sind Übelkeit und/oder Erbrechen in ca. $50 \%$ und Stauungspapillen in ca. $40 \%$.

\section{Risikofaktoren für die Entstehung von Kopfschmerzen bei Hirntumoren}

In einer epidemiologischen Studie konnten vier Faktoren identifiziert werden, die Risikofaktoren für die Ausprägung von Kopfschmerzen bei Hirntumoren sind (14):

- Ein erhöhter intrakranieller Druck geht in über $80 \%$ der Patienten mit Kopfschmerzen einher.

- Die Lokalisation des Tumors spielt eine wichtige Rolle. $82 \%$ der Patienten mit infratentoriellen und 75\% der Patienten mit leptomeningealen Tumoren hatten Kopfschmerzen.

- Die Größe des Tumors und vor allem die Mittellinienverlagerung sind ebenfalls mit der Ausprägung von Kopfschmerzen signifikant positiv korreliert.

- Demgegenüber zeigt die Größe des Ödems einen geringer ausgeprägten $\mathrm{Zu}$ sammenhang mit den Kopfschmerzen, hierzu existieren jedoch auch widersprüchliche Daten (15).

Wenn idiopathische Kopfschmerzen bereits vor dem Hirntumor bestanden haben, ist das Risiko für die Entstehung von Kopfschmerzen durch den Hirntumor größer (16). In vielen Fällen verstärken sich dann durch den Hirntumor die vorbestehenden Kopfschmerzen. Dieses Phänomen ist auch aus pathophysiologischer Sicht von Interesse, da es nahelegt, dass durch einen Hirntumor nicht nur über die direkte raumfordernde Wirkung, sondern auch über andere Mechanismen, die bei idiopathischen Kopfschmerzen eine wichtige Rolle spielen (wie z. B. die Aktivierung des trigeminovaskulären Systems), Kopfschmerzen verursacht oder ausgelöst werden können.

\section{Verbindung mit idiopathischen Kopfschmerzen}

Kopfschmerzen aufgrund von Hirntumoren bieten am häufigsten das Bild eines Kopfschmerzes vom Spannungstyp (17). So erfüllen 77\% aller Patienten mit Hirntumoren und Kopfschmerzen die Kriterien für einen solchen Kopfschmerz und immerhin noch 9\% für eine Migräne (14). In dieser Fallserie konnte kein Zusammenhang zwischen der Lokalisation des Tumors und der Art der Kopfschmerzen hergestellt werden.

In der Literatur sind mehrfach Fallberichte publiziert worden über eine Assoziation zwischen verschiedenen Arten von Hirntumoren und Migräne ohne und mit Aura. Hier ist letztlich die Frage nicht abschließend gelöst, ob es sich um eine rein symptomatische Variante der Migräne handelt oder ob der Hirntumor als auslösender Faktor für Migräneattacken auf Grundlage einer vorbestehenden Veranlagung gewertet werden muss. Bemerkenswerterweise können die migräneartigen Kopfschmerzen, die in Verbindung mit einem Hirntumor aufgetreten sind, gut auf migräneprophylaktische Medikamente ansprechen.

Auch für verschiedene Kopfschmerzformen aus dem Kreis der trigemino-autono- 


\begin{tabular}{|c|c|c|}
\hline Quelle & Kopfschmerzart & Tumor \\
\hline Cala et al. (28) & Migräne (mit Teichopsien) & Glioblastoma multiforme \\
\hline Pepin (29) & Migräne mit Aura & $\begin{array}{l}\text { parietal gelegene } \\
\text { Metastase }\end{array}$ \\
\hline Schlake et al. (30) & Migräne & Meningeom \\
\hline Verma et al. (31) & Migräne mit Aura & $\begin{array}{l}\text { occipital gelegener } \\
\text { Tumor }\end{array}$ \\
\hline $\begin{array}{l}\text { de Angelis et al. } \\
\text { (32) }\end{array}$ & Clusterkopfschmerz & primäres ZNS-Lymphom \\
\hline Greve et al. (33) & Clusterkopfschmerz & Akustikusneurinom \\
\hline Hannerz (34) & Clusterkopfschmerz & Meningeom parasellär \\
\hline Taub et al. (35) & Clusterkopfschmerz & $\begin{array}{l}\text { Meningeom am } \\
\text { Tentorium }\end{array}$ \\
\hline Milos et al. (36) & Clusterkopfschmerz & Hypophysenadenom \\
\hline Tajti et al. (37) & Clusterkopfschmerz & Metastase \\
\hline $\begin{array}{l}\text { Porta-Etessam et } \\
\text { al. (38) }\end{array}$ & Clusterkopfschmerz & Prolaktinom \\
\hline Edvardsson (39) & Clusterkopfschmerz & Hypophysenadenom \\
\hline Medina (40) & Paroxysmale Hemikranie & $\begin{array}{l}\text { Gangliozytom in der } \\
\text { Sella }\end{array}$ \\
\hline Vijayan (41) & Paroxysmale Hemikranie & $\begin{array}{l}\text { Gangliozytom in der } \\
\text { Sella }\end{array}$ \\
\hline Massiou et al. (42) & SUNCT-Syndrom & Prolaktinom \\
\hline $\begin{array}{l}\text { Kutschenko et al. } \\
\text { (43) }\end{array}$ & SUNCT-Syndrom & Meningeom \\
\hline Musuka et al. (44) & SUNCT-Syndrom & Hpyophysenadenom \\
\hline
\end{tabular}

Tab. 3

Fallberichte über die Assoziation von idiopathischen Kopfschmerzen mit einem Hirntumor. men Kopfschmerzen und der idiopathischen Kopfschmerzen ohne strukturelle Läsion sind Assoziationen zwischen diesen Kopfschmerzen und Hirntumoren beschrieben worden (18). Insbesondere beim Clusterkopfschmerz (19) und beim sog. benignen Hustenkopfschmerz und beim benignen Anstrengungskopfschmerz $(3,20)$ sind intrakranielle Raumforderungen als Auslöser in ca. 10\% der Fälle beschrieben worden. In Tabelle 3 sind typische, in der Literatur beschriebene Fälle einer symptomatischen Form von Migräne oder trigeminoautonome Kopfschmerzen aufgelistet.

Schließlich soll noch darauf hingewiesen werden, dass idiopathische Kopfschmerzen, insbesondere Migräne, keinen Risikofaktor für die Entstehung eines Hirntumors darstellen (21).

\section{Kopfschmerzen bei Schädelbasismetastasen}

Bei einem Befall der Schädelbasis mit nicht hirneigenen Tumoren kann es ebenfalls zu charakteristischen Kopfschmerzen kommen. Greenberg et al. (22) haben dabei fünf Hauptlokalisationen differenziert.

- Metastasen der Orbita, z. B. bei Karzinomen der Mamma, Lunge und Prostata. Hier kommt es zu einem supraorbitalen Kopfschmerz, der häufig von Bulbusbewegungsstörungen begleitet ist.

- Metastasen im Bereich der Sella. Hier wird häufig der Sinus cavernosus mit betroffen. Es entsteht ein einseitiger frontaler Kopfschmerz verbunden mit einer Schwellung der Orbita. Auch hier kann es zu Bulbusbewegungsstörungen kommen.

- Metastasen im Bereich des Ganglion Gasseri. Zu solchen Metastasen kann es durch Karzinome der Mamma, der Lunge und des Halses (z. B. Pharynx) kommen. Betroffen ist vor allem der N. trigeminus, sodass die Schmerzmanifestation in den verschiedenen Versorgungsgebieten dieses Nerven vorkommen kann.

- Metastasen im Bereich des Foramen jugulare. Hier kommt es zu einem Kopfschmerz hinter den Ohren und nuchal. Begleitend zum Kopfschmerz können Schluckstörungen und Heiserkeit auftreten. Durch die intrakranielle Druckerhöhung kann es auch zu einer Stauungspapille kommen.

- Metastasen der okzipitalen Kondylen. Hierbei kommt es zu einem einseitigen okzipitalen Schmerz, der sich bei Nackenbeugung verstärkt. Begleitend können Dysarthrie, Schluckstörungen und selten auch eine Zungenatrophie auftreten.

\section{Diagnostik}

Sinnvoll und dringend indiziert ist eine Bildgebung dann, wenn bei einem Patienten mit einem bekannten Karzinom (insbesondere Lunge, Mamma, Prostata, Melanom) bislang unbekannte Kopfschmerzen auftreten, bislang bekannte Kopfschmerzen sich verstärken oder Kopfschmerzen neu in Zusammenhang mit fokalneurologischen Symptomen auftreten. Dies ist auch einer der wesentlichen Gründe, warum bei einer Erstdiagnostik von Kopfschmerzen eine körperlich-neurologische Untersuchung unerlässlich ist. Die Bildgebung kann initial aus einer kranialen Computertomografie (CCT) bestehen. Zwingend ist bei dem Verdacht auf einen Hirntumor immer die Gabe von Kontrastmittel, da kleinere hirneigene Tumore und viele Metastasen sich häufig nur so auch zweifelsfrei darstellen lassen. Bei Verdacht auf Schädelbasismetastasen oder auf Tumore der Gesichtsstrukturen (z. B. der Nasennebenhöhlen) kann auch ein Knochenfenster angezeigt sein, um z. B. die Invasion eines Tumors in den intrakraniellen Raum durch den Knochen hindurch zu beurteilen. Goldstandard ist jedoch die Kernspintomografie (MRT) mit Gabe von Gadolinium, da diese auch kleinere Tumore als das CCT aufdecken 
und Tumore der hinteren Schädelgrube und des Hirnstamms besser darstellen kann. Weiterhin kann man in der MRT auch die Meningen beurteilen, die bei einer Meningeosis verdickt sein können und Kontrastmittel aufnehmen. Schließlich kann in einer MRT auch besser die Beziehung des Tumors zu anderen intrakraniellen Strukturen beurteilt werden. Dies ist häufig wichtig, da die Kopfschmerzen im Allgemeinen über diese Beziehungen (und nicht allein durch das Neoplasma des Tumors) entstehen und somit die Therapieplanung (operativ oder strahlentherapeutisch) durchgeführt werden kann.

Neurophysiologische Untersuchungen sind für die Diagnostik von Hirntumoren im Allgemeinen nicht notwendig. Allenfalls kann das EEG einen Herdbefund aufdecken, in typischen Fällen mit einer Phasenumkehr, die den Schwerpunkt der intrakraniellen Raumforderung lokalisiert. In manchen Fällen, insbesondere bei Verdacht auf Meningeosis, kann eine Lumbalpunktion mit Liquoranalyse (z. B. Tumorzellen im Liquor) sinnvoll sein. Die Lumbalpunktion sollte immer nach der zerebralen Bildgebung erfolgen, da dann zum einen ein erhöhter Hirndruck ausgeschlossen ist und es zum anderen durch eine Lumbalpunktion zu einer Kontrastmittelanreicherung der Meningen kommen kann, die dann mit einer Meningeosis verwechselt werden kann.

\section{Therapeutische Prinzipien}

Die Therapie von Kopfschmerzen bei Hirntumoren sollte natürlich in erster Linie die Beseitigung der Ursache, nämlich der hirneigenen Tumoren, der Meningeosis oder der Metastasen zum Ziel haben. Dies wird jedoch nur in den seltensten Fällen gelingen. Außerdem verschwindet der Kopfschmerz nicht notwendigerweise nach der Tumorentfernung, so z. B. bei Tumoren der Sella-Region (23). Die eigentliche Hirntumortherapie ist auch nicht Gegenstand dieser Übersicht. Es kann auch primär zur Schmerztherapie sinnvoll sein, einen Shunt des Ventrikelsystems (extern oder ventrikuloperitoneal) anzulegen, um eine rasche intrakranielle Druckentlastung herbeizuführen.

Tab. 4

Pragmatische Stufentherapie zur Behandlung von Kopfschmerzen aufgrund von Hirntumoren.

\begin{tabular}{|l|l|}
\hline Schmerzintensität & Therapie \\
\hline Leichte Schmerzen & $\begin{array}{l}\text { Paracetamol } 3 \times 500 \mathrm{mg} \text { und/oder } \\
\text { Metamizol } 3 \times 500 \mathrm{mg}\end{array}$ \\
\hline Mittlere Schmerzen & $\begin{array}{l}\text { Tramadol bis } 3 \times 200 \mathrm{mg} \text { und/oder Tilidin } \\
\text { bis } 3 \times 200 \mathrm{mg}\end{array}$ \\
\hline Starke Schmerzen & $\begin{array}{l}\text { Morphin bis } 4 \times 100 \mathrm{mg} \text { oder Methadon } \\
\text { bis 3 } 7,5 \mathrm{mg}\end{array}$ \\
\hline Adjuvante Schmerztherapie & $\begin{array}{l}\text { Amitriptylin bis } 150 \text { mg mit abendlichem } \\
\text { Schwerpunkt; Dexamethason } 3 \times 4 \mathrm{mg} \\
\text { bis } 4 \times 20 \mathrm{mg}\end{array}$ \\
\hline
\end{tabular}

Kopfschmerzen bei Hirntumoren gehören zu den ganz wenigen Indikationen, bei denen Kopfschmerzen auch eine Indikation für Opioide darstellen. Es gibt bis heute keine kontrollierten Studien, die belegen würden, welches Therapieregime zur Behandlung bei Kopfschmerzen aufgrund von Hirntumoren am geeignetsten ist. Daher beruhen die folgenden Empfehlungen auf Lehrbuchpublikationen und Analogieschlüssen.

Bei leichten Schmerzen sollte Paracetamol in regelmäßiger Dosierung gegeben werden (z. B. 3 x $500 \mathrm{mg}$ pro Tag), wenn eine Veränderung des Blutbilds nicht besteht kann auch Metamizol eingesetzt werden. Acetylsalicylsäure und andere NSAR sind wegen ihres Einflusses auf das Gerinnungssystem häufig nicht sinnvoll, insbesondere dann nicht, wenn noch ein operativer Eingriff bevorsteht. Sollte Paracetamol nicht ausreichend wirksam sein, sollte zusätzlich noch ein schwach wirksames Opioid eingesetzt werden (z. B. Codein, Tramadol, Tilidin in Kombination mit Naloxon). Als letzte Eskalationsstufe kann dann ein stark wirksamen Opioid eingesetzt werden (z B. Morphin, Polamidon, Fentanyl). Opioide können in seltenen Fällen den intrakraniellen Druck steigern, dieses Risiko ist für die ersten Tage der Therapie zu beachten, muss aber in der Abwägung häufig in Kauf genommen werden. Wichtig ist auch die Applikationsform des Opioids (z. B. als Pflaster, in retardierter Form). $>$ Tabelle 4 gibt eine pragmatische Übersicht über die Stufentherapie bei Kopfschmerzen aufgrund von Hirntumoren. Gerade bei der Behandlung mit Opioiden ist dabei jedoch zu bedenken, dass die Dosierung individuell erfolgen muss (d. h. geringst mögliche Dosis des Opiats für eine maximale Schmerzlin- derung unter Beachtung der möglichen Nebenwirkungen wie z. B. Obstipation und Benommenheit).

Als adjuvante Substanz in der Schmerztherapie kann ein trizyklisches Antidepressivum eingesetzt werden. Dies kommt vor allem in Betracht, wenn eine neuropathische Schmerzkomponente auftritt, z. B. aufgrund einer peripheren Affektion des N. trigeminus. Im letzteren Fall kann auch eine membranstabilisierende Substanz wie z. B. Carbamazepin oder Gabapentin gegeben werden. Eine weitere adjuvante Maßnahme in der Schmerztherapie von Kopfschmerzen aufgrund von Hirntumoren ist in den vielen Fällen mit erhöhtem intrakraniellen Druck die Gabe eines Steroids. Diese Gabe kann sich manchmal schon innerhalb weniger Stunden als deutlich schmerzlindernd erweisen, außerdem können fokalneurologische Symptome durch Nervenkompression abklingen. Insbesondere bei Nachweis einer perifokalen Ödems aufgrund des Hirntumors ist ein Steroid indiziert (z. B. Dexamethason initial $40 \mathrm{mg}$ bis $100 \mathrm{mg}$, dann von $3 \times 4 \mathrm{mg}$ bis $4 \times 20 \mathrm{mg}$ dosierbar). Steroide sollten nicht gegeben werden bei Verdacht auf ein Lymphom, bevor eine Hirnbiopsie durchgeführt worden ist, da Lymphome gut auf Steroide ansprechen. Schließlich kann auch die Strahlentherapie eine adjuvante Maßnahme der Schmerztherapie bei Kopfschmerzen sein, wobei allerdings in den ersten Phasen der Bestrahlung durch ein vermehrtes Hirnödem eine Zunahme von Kopfschmerzen auftreten kann. Daher sollte gerade die Strahlentherapie immer in Kombination mit der Gabe von Steroiden durchgeführt werden. 


\section{Interessenkonflikt}

Der Autor erklärt keine Interessenkonflikte in Zusammenhang mit diesem Artikel.

\section{Literatur}

1. Hopkins A. Headache: Problems in diagnosis and management. London: Saunders 1988.

2. Evers S. Kopfschmerz bei Hirntumoren. In: Diener HC (Hrsg.). Kopfschmerzen. Referenzreihe Neurologie. Stuttgart: Thieme 2003: 140-146.

3. Forsyth PA, Posner JB. Intracranial neoplasms. In: Olesen J, Tfelt-Hansen P, Welch KMA (eds.) The headaches. Philadelphia: Lippincott Williams \& Wilkins 2000, 849-859.

4. Suwanwela N, Phanthumchinda K, Kaoropthum S. Headache in brain tumor: a cross-sectional study. Headache 1994; 34: 435-438.

5. Iversen HK, Strange P, Sommer W, Tjalve E. Brain tumour headache related to tumour size, histology and location. Cephalalgia 1987; 7 Suppl 6: 394-395.

6. Ludwig CL, Smith MT, Godfrey AD, Armbrustmacher VW. A clinicopathological study of $323 \mathrm{pa}-$ tients with oligodendrogliomas. Ann Neurol 1986; 19: 15-21.

7. Jaeckle KA. Causes and management of headaches in cancer patients. Oncology 1993; 7: 27-31.

8. Vazquez-Barquero A, Ibanez FJ, Herrera S, Izquierdo JM, Berciano J, Pascual J. Isolated headache as the presenting clinical manifestation of intracranial tumors: a prospective study. Cephalalgia 1994; 14: 270-272.

9. Clementi M, Battistella PA, Rizzi L, Boni S, Tenconi R. Headache in patients with neurofibromatosis type 1. Headache 1996; 36: 10.

10. Rushton JG, Rooke ED. Brain tumor headache. Headache 1962; 2: 147-152.

11. Lavyne MH, Patterson RH. Headache and brain tumor. In: Dalessio DJ (Hrsg) Wolff's headache and other head pain. New York: Oxford University Press 1987, 343-349.

12. Dunlop RJ, Campbell CW. Cytokines and advanced cancer. J Pain Symptom Manage 2000; 20: 214-232.

13. Evans RW. Diagnostic testing for the evaluation of headaches. Neurol Clin 1996; 14: 1-26.

14. Forsyth PA, Posner JB. Headaches in patients with brain tumors: a study of 111 patients. Neurology 1993; 43: 1678-1683.

15. Pfund Z, Szapary L, Jaszberenyi O, Nagy F, Czopf J. Headache in intracranial tumors. Cephalalgia 1999; 19: 787-790.
16. Schankin CJ, Ferrari U, Reinisch VM, Birnbaum T, Goldbrunner R, Straube A. Characteristics of brain tumour-associated headache. Cephalalgia 2007; 27: 904-911.

17. Nelson S, Taylor LP. Headaches in brain tumor patients: primary or secondary? Headache 2014; 54: 776-785.

18. Levy MJ, Matharu MS, Meeran K, Powell M, Goadsby PJ. The clinical characteristics of headache in patients with pituitary tumours. Brain 2005; 128 : 1921-1930.

19. Sörös P, Evers S. Symptomatischer Clusterkopfschmerz. Klinik, Diagnostik und mögliche Pathogenese. Nervenheilkunde 2001; 20: 370-374.

20. Pascual J, Iglesias F, Oterino A, Vazquez-Barquero A, Berciano J. Cough, exertional, and sexual headaches: an analysis of 72 benign and symptomatic cases. Neurology 1996; 46: 1520-1524.

21. Kurth T, Buring JE, Rist PM. Headache, migraine and risk of brain tumors in women: prospective cohort study. J Headache Pain 2015; 16: 501.

22. Greenberg HS, Deck MD, Vikram B, Chu FC, Posner JB. Metastasis to the base of the skull: clinical findings in 43 patients. Neurology 1981; 31: 530-537.

23. Siegel S, Weber Carneiro R, Buchfelder M, Kleist B, Grzywotz A, Buslei R, Bingel U, Brabant G, Schenk T, Kreitschmann-Andermahr I. Presence of headache and headache types in patients with tumors of the sellar region-can surgery solve the problem? Results of a prospective single center study. Endocrine 2017; 56: 325-335.

24. Childhood Brain Tumor Consortium (CBTC). The epidemiology of headache among children with brain tumor. J Neurooncol 1991; 10: 31-46.

25. Schankin CJ, Reifferscheid AK, Krumbholz M, Linn J, Rachinger W, Langer S, Sostak P, Arzberger T, Kretzschmar H, Straube A. Headache in patients with pituitary adenoma: clinical and paraclinical findings. Cephalalgia 2012; 32: 1198-1207.

26. Russo M, Villani V, Taga A, Genovese A, Terrenato I, Manzoni GC, Servadei F, Torelli P, Pace A. Headache as a presenting symptom of glioma: A cross-sectional study. Cephalalgia 2017; 57(4): 663-664.

27. Guillamo JS, Monjour A, Taillandier L, Devaux B, Varlet P, Haie-Meder C, Defer GL, Maison P, Mazeron JJ, Cornu P, Delattre JY. Brainstem gliomas in adults: prognostic factors and classification. Brain 2001; 124: 2528-2539.

28. Cala LA, Mastaglia FL. Computerized axial tomography findings in a group of patients with migrainous headaches. Proc Aust Assoc Neurol 1976 13: 35-41.

29. Pepin EP. Cerebral metastasis presenting as migraine with aura. Lancet 1990; 336: 127-128.
30. Schlake HP, Grotemeyer KH, Husstedt IW, Schuierer G, Brune GG. Symptomatic migraine: intracranial lesions mimicking migrainous headache - a report of three cases. Headache 1991; 31: 661-665.

31. Verma A, Rosenfeld V, Forteza A, Sharma KR. Occipital lobe tumor presenting as migraine with typical aura. Headache 1996; 36: 49-52.

32. De Angelis LM, Payne R. Lymphomatous meningitis presenting as atypical cluster headache. Pain 1987; 30: 211-216.

33. Greve E, Mai J. Cluster headache-like headaches: a symptomatic feature? A report of three patients with intracranial pathological findings. Cephalalgia $1988 ; 8: 79-82$.

34. Hannerz J. A case of parasellar meningioma mimicking cluster headache. Cephalalgia 1989; 9: 265-269.

35. Taub E, Argoff CE, Winterkorn JMS, Milhorat TH. Resolution of chronic cluster headache after resection of a tentorial meningioma: case report. Neurosurgery 1995; 37: 319-321.

36. Milos P, Havelius U, Hindfelt B. Clusterlike headache in a patient with a pituitary adenoma. With a review of the literature. Headache 1996; 36: 184-188.

37. Tajti J, Sas K, Szok D, Voros E, Vecsei L. Clusterlike headache as a first sign of brain metastases of lung cancer. Headache 1996; 36: 259-260.

38. Porta-Etessam J, Ramos-Carrasco A, Berbel-Garcia A, Martinez-Salio A, Benito-Leon J. Clusterlike headache as first manifestation of a prolactinoma. Headache 2001; 41: 723-725.

39. Edvardsson B. Cluster headache associated with a clinically non-functioning pituitary adenoma: a case report. J Med Case Rep 2014; 8: 451.

40. Medina JL. Organic headaches mimicking chronic paroxysmal hemicrania. Headache 1992; 32: 73-74.

41. Vijayan N. Symptomatic chronic paroxysmal hemicrania. Cephalalgia 1992; 12: 111-113.

42. Massiou H, Launay JM, Levy C, El Amrani M, Emperauger B, Bousser MG. SUNCT syndrome in two patients with prolactinomas and bromocriptine-induced attacks. Neurology 2002; 58: 1698-1699.

43. Kutschenko A, Liebetanz D. Meningioma causing gabapentin-responsive secondary SUNCT syndrome. J Headache Pain 2010; 11: 359-361.

44. Musuka TD, Edis RH, Kermode AG. Short-lasting unilateral neuralgiform headache with conjunctival injection and tearing caused by a pituitary adenoma. J Clin Neurosci 2013; 20: $1180-1181$ 


\title{
Zertifizierte Fortbildung
}

\section{in Zusammenarbeit mit der Bayerischen Landesärztekammer}

\author{
Fragen zum Thema „Kopfschmerzen bei Hirntumoren”
}

1. Wie groß ist die generelle Wahrscheinlichkeit, dass ein erstmaliger Kopfschmerz durch einen Hirntumor verursacht wird?

a) $20 \%$

b) $10 \%$

c) $5 \%$

d) unter $1 \%$

e) Hirntumore verursachen keine erstmaligen Kopfschmerzen.

2. Welche Aussage zur Prävalenz von Kopfschmerzen bei verschiedenen Hirntumoren ist richtig?

a) Kopfschmerzen bei supratentoriellen Hirntumoren sind seltener als bei infratentoriellen Hirntumoren.

b) Extrazerebrale Tumoren führen häufiger zu Kopfschmerzen als intrazerebrale.

c) Hochmaligne Gliome führen seltener zu Kopfschmerzen als niedriggradige Astrozytome.

d) Nasopharyngeale Karzinome führen sehr selten zu Kopfschmerzen.

e) Kopfschmerzen treten insgesamt nur bei ca. $20 \%$ aller Patienten mit einem Hirntumor auf.

3. Was ist das häufigste Erstsymptom bei niedriggradigen Astrozytomen?

a) Kopfschmerzen

b) Halbseitenlähmung

c) Hirnnervenausfälle

d) Bewusstseinstrübung

e) Epileptische Anfälle

4. Welcher der folgenden Mechanismen ist typischerweise nicht für Kopfschmerzen bei Hirntumoren ursächlich?

a) Direkte Reizung schmerzsensibler Strukturen in den Gefäßen.

b) Reizung der Subarachnoidea.

c) Funktionsverlust von Neuronen.

d) Raumforderung und damit verbundene Ödembildung.

e) Behinderung des Liquorabflusses und damit Hydrocephalus internus.
5. Welche Aussage zur Semiologie von Kopfschmerzen bei Hirntumoren ist richtig?

a) Kopfschmerzen aufgrund von Hirntumoren sind in ihrer Intensität nicht konstant.

b) Ca. 2\% der Patienten hat morgendliche Kopfschmerzen mit Erbrechen.

c) Bei ca. $80 \%$ erweckt der Kopfschmerz die Patienten morgens aus dem Schlaf.

d) Ein Valsalva-Manöver führt bei ca. zwei Drittel zu einer vorübergehenden Verstärkung der Kopfschmerzen.

e) Die Lokalisation von Kopfschmerzen aufgrund von Hirntumoren lässt in der Regel Rückschlüsse auf die Lokalisation des Hirntumors zu.

6. Welche Aussage zu Begleitsymptomen von Kopfschmerzen bei Hirntumoren ist richtig?

a) Häufig tritt Kopfschmerz als einziges Symptom eines Hirntumors auf.

b) Übelkeit und/oder Erbrechen treten in ca. $10 \%$ der Patienten auf.

c) Stauungspapillen liegen in ca. $80 \%$ vor.

d) Kopfschmerzen ohne weitere subjektive Symptome haben ca. 10\% aller Patienten.

e) Eine visuelle Aura wird häufig beschrieben.

7. Was ist kein Risikofaktor für die Entstehung von Kopfschmerzen bei Hirntumoren?

a) Erhöhter intrakranieller Druck

b) Infratentorielle Lokalisation

c) Mittellinienverlagerung durch den Tumor

d) Vorbestehende idiopathische Kopfschmerzen

e) Niedriger Liquordruck

8. Welche Aussage zu Kopfschmerzen bei Hirntumoren ist richtig?

a) Eine Migräne ist ein Risikofaktor für die Entstehung von Hirntumoren.

b) Hirntumoren können trigemino-autonome Kopfschmerzen imitieren.

c) Hirntumore können keine migräneartigen Kopfschmerzen imitieren.

d) Intrakranielle Metastasen können nicht zu Kopfschmerzen führen.
CME-Fortbildung online

Die Teilnahme an der CME-Fortbildung ist für 12 Monate ausschließlich online möglich. Zur Anmeldung gehen Sie bitte auf cme.schattauer.de. Dort erfahren Sie auch den genauen Teilnahmeschluss. Es ist immer nur eine Antwort pro Frage zutreffend. Als Leser der Nervenheilkunde nehmen Sie kostenlos am CME-Programm teil. Je nach CME-Fortbildung erhalten Sie bis zu vier Fortbildungspunkte. Weitere Informationen zur Anmeldung und Registrierung finden Sie unter cme.schattauer.de. Sie erhalten bei inhaltlichen und technischen Fragen tutoriellen Support.

e) Hustenkopfschmerz und benigner Anstrengungskopfschmerz können nicht symptomatisch durch Hirntumore ausgelöst werden.

9. Welche Aussage zur Diagnostik bei Kopfschmerzen bei Hirntumoren ist richtig?

a) Zwingend ist bei dem Verdacht auf einen Hirntumor die Gabe von Kontrastmittel bei MRT oder CCT.

b) Bei Verdacht auf Schädelbasismetastasen genügt eine Nativ-Röntgenaufnahme.

c) Tumore der hinteren Schädelgrube und des Hirnstamms können besser im CCT gesehen werden.

d) Neurophysiologische Untersuchungen wie EEG sind zwingend notwendig für die Diagnostik von Hirntumoren.

e) Eine Lumbalpunktion sollte immer vor der zerebralen Bildgebung erfolgen.

10. Welche Aussage zur konservativen Therapie von Kopfschmerzen bei Hirntumoren ist richtig?

a) Opioide haben keinen Stellenwert in der Schmerztherapie bei Hirntumoren.

b) Steroide sind in der Schmerzlinderung bei Hirntumoren wirkungslos.

c) Bei Schmerzen im Bereich der Hirnnerven können Membranstabilisatoren eingesetzt werden.

d) Trizyklische Antidepressiva werden nicht eingesetzt.

e) Acetylsalicylsäure ist immer Analgetikum der ersten Wahl bei Hirntumoren. 